\title{
Exo-Electron Emission from Metal Surfaces with the Progress of Fatigue Damage*
}

\author{
By Kenjiro Komai**
}

\begin{abstract}
The exo-electron emission from metal surfaces subjected to repeated stresses has been studied using an electron-multiplier counting system, and the environmental effects on fatigue strength are discussed from the viewpoint of exo-electron emission.

The exo-electrons from metal surfaces are sharply increased by repeated stresses and take a maximum value at a fixed number of cycles $\left(N_{\mathrm{emax}}\right)$. Thereafter the number of emitted electrons gradually decreases and becomes almost constant. The decay of the emission rate after termination of stress repetitions is very fast, its half life being shorter than $1 \mathrm{~s}$. The decay behaviors at cycle numbers before and after $N_{\text {emax }}$ are different; the decay is markedly fast at the beginning of fatigue cycles. The decay of the emission rate from a surface subjected to fatigue damage is, however, smaller than that from a mechanically processed surface. The pulse height distribution of emitted electrons is hardly affected by the progress of fatigue damage. However, sufficient allowances should be made for the role of exo-electrons in the environmental effects on the fatigue strength, in view of the energy height of exo-electrons. The number of exo-electrons per cycle increases markedly with increasing stress cycle frequency.
\end{abstract}

(Received July 20, 1977)

\section{Introduction}

A freshly created metal surface emits electrons without any apparent energy supply, which is a well-known phenomenon as "exoelectron emission" (The Kramer effect ${ }^{(\mathbf{1})}$ ). The exo-electron emission is observed from metal surface subjected to mechanical proces$\operatorname{sing}^{(2)}$, repeated stresses ${ }^{(3) \sim(5)}$ and chemical reaction. However, the role of the exo-electron emission in fatigue, delayed failure, and wear and friction has remained unexplained.

Since the exo-electron emission is affected by the defect density, magnitude of strain, extent of oxidation and so forth, the exoelectron emission may be a powerful means to clarify the change of metal surfaces with the progress of fatigue damage. Grunberg ${ }^{(6)}$ has reported that the electrons emitted from freshly cutted metal surfaces form hydrogen peroxide from the environmental water and

* Published in J. Japan Inst. Metals (in Japanese), 40 (1976), 964.

** Department of Mechanical Engineering, Faculty of Engineering, Kyoto University, Kyoto 606, Japan.

Trans. JIM oxygen. The autoradiography by Grosskreutz ${ }^{(7)}$ also has showed an ability of the slip band induced by fatigue cycles to blacken a photographic film, which is known as the Rassel effect. Consequently sufficient allowances should be made for the role of exo-electrons in the environmental effects on fatigue strength.

In the present study, the exo-electrons emitted from metal surfaces subjected to repetitional stresses were measured by using an electron-multiplier counting system, and the behavior of the exo-electron emission with the progress of fatigue damage was discussed to elucidate the environmental effects on the fatigue strength.

\section{Experimental Procedure}

The material tested was a corrosion-resistant aluminum alloy with the chemical composition shown in Table 1. The shape and dimensions of smooth specimens are shown in Fig. 1. The specimens were polished by \#2000 emery paper after final machining, and then annealed in vacuum at $653 \mathrm{~K}$ for $1.5 \mathrm{~h}$. Just prior to the fatigue test the specimen was electrochemically polished.

1978 Vol. 19 
Table 1 Chemical composition of test material. ( $\%)$

\begin{tabular}{cccccccc}
\hline $\mathrm{Mg}$ & $\mathrm{Mn}$ & $\mathrm{Cr}$ & $\mathrm{Cu}$ & $\mathrm{Si}$ & $\mathrm{Fe}$ & $\mathrm{Zn}$ & $\mathrm{Al}$ \\
\hline 5.25 & 0.11 & 0.10 & 0.01 & 0.07 & 0.11 & 0.02 & $\mathrm{bal}$ \\
\hline
\end{tabular}

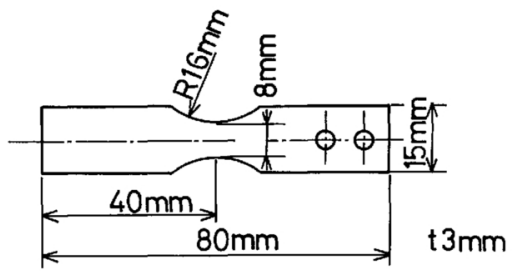

Fig. 1 Shape and dimensions of specimens.

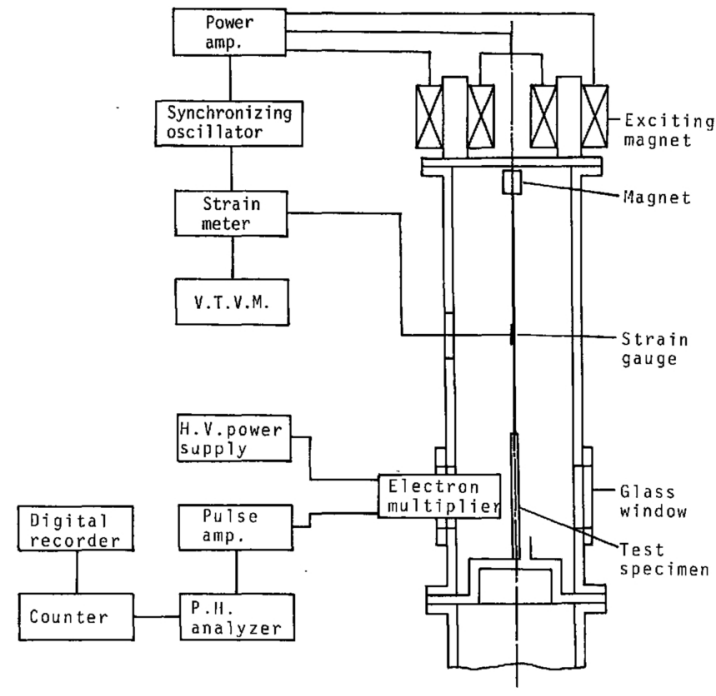

Fig. 2 Testing apparatus.

The fatigue test was carried out by a planebending fatigue testing machine of the resonant type as shown in Fig. 2. The specimen was subjected to repeated stresses by attractive and repulsive forces of exciting magnets. The resonant frequency detected by a strain gauge on the extension rod was fed back to synchronizing oscillator to persuit automatically the resonant frequency of the specimen. Repeated stresses were thus stabilized throughout the fatigue test. The stress cycle frequency was in $13.9 \sim 14.5 \mathrm{~Hz}$, though it decreased slightly with stress cycles. Repeated stresses at $0.038 \sim$ $0.5 \mathrm{~Hz}$ were also applied, where a special driving device was incorporated into the testing apparatus.
The block diagram of an electron-multiplier counting system is also shown in Fig. 2. Electrons emitted from specimen surfaces were collected and amplified by an electronmultiplier which was located in the neighborhood of the specimen. Thereafter the electrons were processed through a pulse amplifier, a pulse-height analyzer and finally counted by a digital counter. The electronmultiplier was a box and grid electrode type of 20-stage dynodes. Its gain was more than $1 \times 10^{9}$ under an applied potential of $4 \mathrm{kV}$ between the first dynode and an anode. The first dynode with a projected area of $12 \times 10$ $\mathrm{mm}$ was located $10 \mathrm{~mm}$ apart from the specimen surface of minimum cross-section and the applied potential between the first dynode and the specimen and the one between the first dynode and an anode were 1 and $4 \mathrm{kV}$, respectively. The threshold levels of the counting system were 10 and $45 \mathrm{mV}$ at the out-put of the electron-multiplier, and the window width to measure a pulse-height distribution of emitted electrons was $20 \mathrm{mV}$.

Electrons emitted with the progress of stress cycles were counted in vacuum at $1 \sim 2 \times 10^{-3}$ $\mathrm{Pa}$ in a chamber made of SUS 304 stainlesssteel after the glass window was covered by a shutter to inhibit the light incidence to the first dynode.

\section{Experimental Results and Discussion}

\section{Exo-electron emissions with the progress of stress cycles}

Variations in exo-electron emission rate with the progress of stress cycles under various stress amplitudes are illustrated in Figs. 3, 4 and 5 . In a case where the threshold level is taken as $10 \mathrm{mV}$, the counted rate of electrons emitted from an electrochemically polished surface is less than several counts per sec before the fatigue test. The rate of emitted electrons is sharply increased immediately after the beginning of the fatigue test and takes a maximum value. Thereafter the counted rate gradually decreases and becomes almost constant. The same emission characteristic was also observed in 1100-0 aluminum in vacuum and in air $^{(4)}$. Under a stress amplitude of 


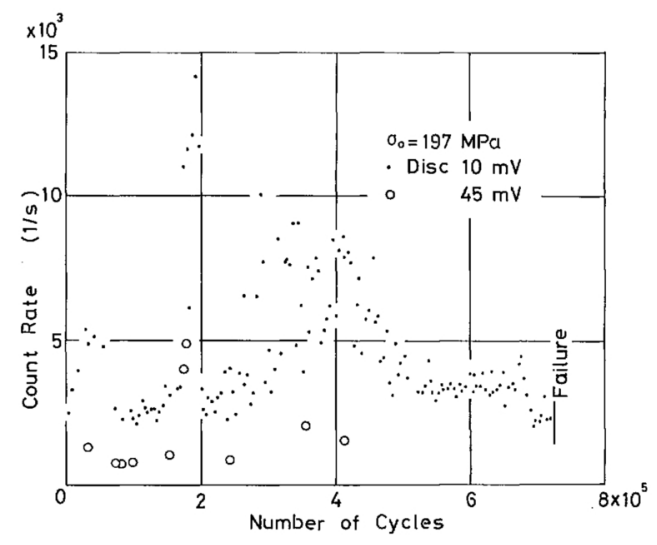

Fig. 3 Variation of the count rate per sec with stress cycles.

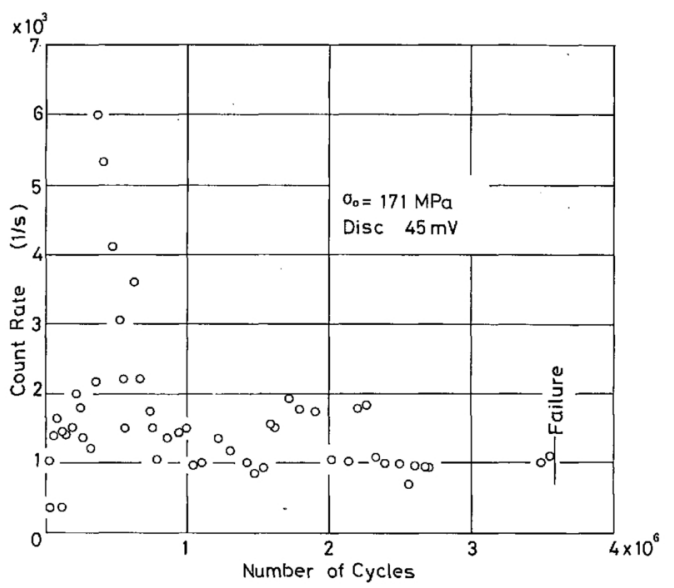

Fig. 4 Variation of the count rate per sec with stress cycles.

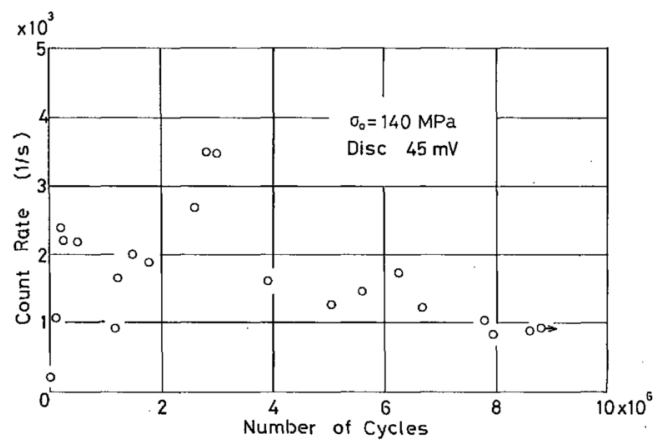

Fig. 5 Variation of the count rate per sec with stress cycles.

$140 \mathrm{MPa}$ where the specimen endures stress cycles of about $9 \times 10^{6}$, there exists also the number of cycles where the counted rate is

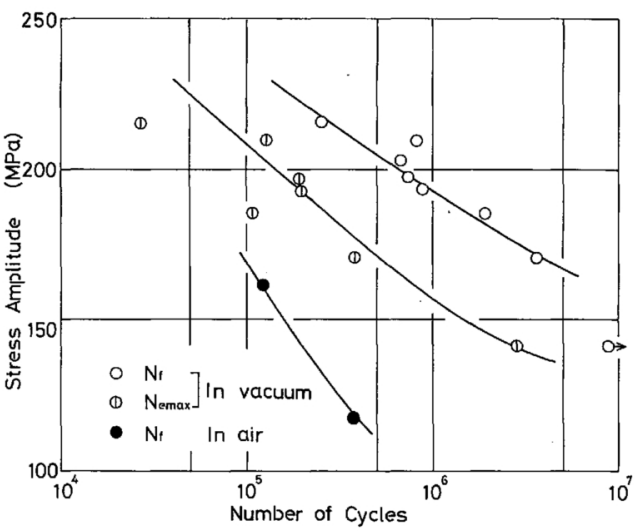

Fig. 6 Relation between number of cycles to failure, $N_{f}$ and number of cycles of maximum count rate, $N_{\text {emax. }}$.

a miximum, as shown in Fig. 5. Figure 6 shows the relation between the number of cycles where the counted rate shows maximum $N_{\text {emax }}$ and that to final fracture $N_{f}$, from which it appears that the ratio of $N_{e m a x}$ to $N_{f}$ becomes greater under a higher stress and a shorter life.

\section{Decay of emitted electrons}

Decay curves of the counted rate after termination of stress repetitions at various stress cycles are shown in Figs. 7 and 8. The ordinate is the fraction of the counted rate devided by the value immediately before the termination of stress cycles, and the abscissa is the time after the termination of stress repetitions on logarithmic scales. The decay of the counted rate is very fast, and its half life is shorter than $1 \mathrm{~s}$. The very fast decay of exo-electrons shows that the increased activity of a specimen surface by repeated stresses decreases in a very short time, which may be related to an important condition of simultaneous action of corrosion and repetitional stresses that causes enhanced damage in corrosion fatigue. The decay is different at cycle numbers before and after $N_{e \max }$ at which the counted rate takes a maximum. The decay is very fast at the beginning of fatigue cycles. The counted rate after a duration greater than $10 \sim 20 \mathrm{~s}$ decreases linearly on logarithmic scales, its slope being constant, -0.33 , except at the beginning of fatigue cycles $(n=2.8 \times$ 


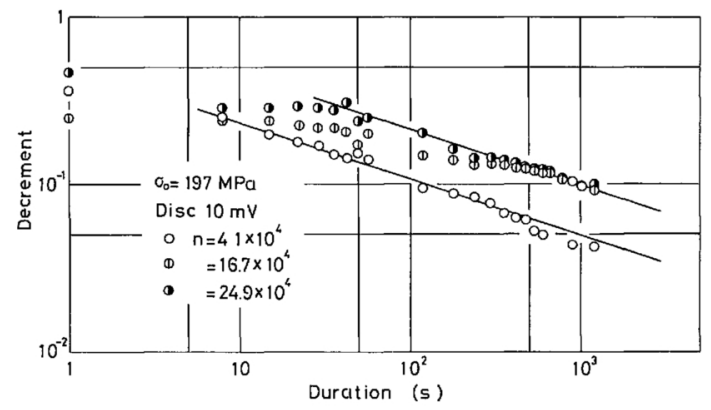

Fig. 7 Decay curves of exo-electron emission.

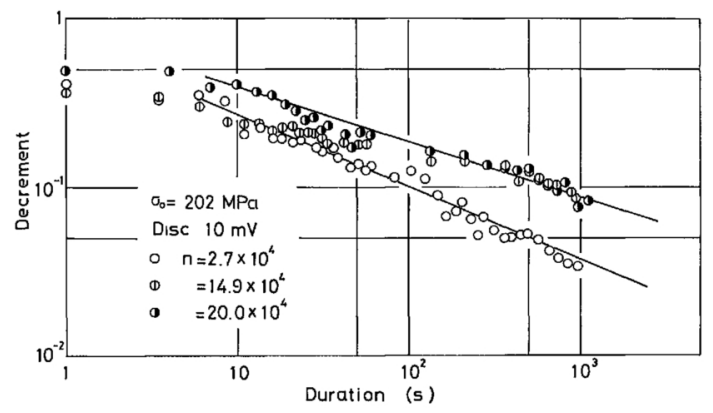

Fig. 8 Decay curves of exo-electron emission.

$10^{4}$ in Fig. 8). The decay curve at $n=24.9 \times$ $10^{4}$ in Fig. 7 almost coincide with the one at $n=20.0 \times 10^{4}$ in Fig. 8 , which proves a good reproducibility of the exo-electron emission.

The slope $(-0.33)$ obtained in Figs. 7 and 8 is much smaller than that $(-1)$ of the emission decay from an aluminum surface abraded by a wire brush ${ }^{(2)}$ or from a metal surface polished by emery paper ${ }^{(8)}$, which shows a more persistent emission characteristic of a surface subjected to fatigue damage than the emission of a mechanically processed surface. On the other hand, it has been reported ${ }^{(5)}$ that the intensity of photoelectron emission by ultraviolet light is quite stable in a high vacuum of $5 \times 10^{-7} \mathrm{~Pa}$ after termination of fatigue cycles. There may be a difference between the emission characteristics of the exo-electron and the photoelectron.

\section{Pulse height distribution}

Figure 9 illustrates a pulse height distribution of emitted electrons at various stress cycles. The ordinate is the frequency of electrons counted in the window width of $20 \mathrm{mV}$, and

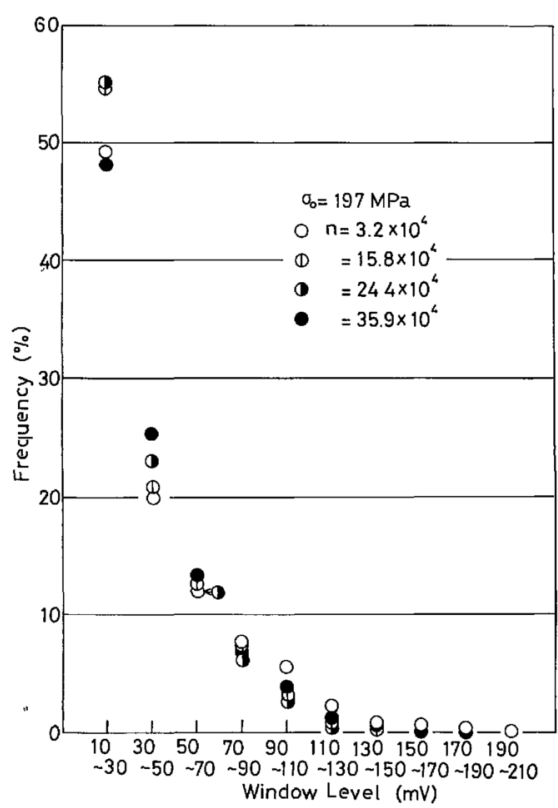

Fig. 9 Pulse height distribution of exo-electrons.

the abscissa the window level of the pulse height analyzer. As is known from the figure the pulse height distribution is independent of fatique damage and an almost invariable pulse height distribution is obtained irrespective of stress cycles.

Since the averaged kinetic energy of emitted electrons from a mechanically processed surface was reported to be $0.2 \sim 2 \mathrm{eV}^{(8)}$, the energy is nearly equal to the activation energy which is necessitated to cause a chemical reaction. Consequently the electron emission from metal surfaces subjected to repeated stresses is considered to play an important role in the environmental fatigue of metals. The influence of oxygen and water vapor in the atmosphere on fatigue strength is different among materials. The damaging effect of water vapor is prominent in the fatigue of aluminum alloy and high-strength steel, which is sometimes explained by hydrogen embrittlement. However, the mechanism has remained unexplained. The bonding energies of $\mathrm{H}-\mathrm{OH}$ and $\mathrm{O}-\mathrm{H}$ are $501 \mathrm{~kJ} / \mathrm{mol}$ and $423 \mathrm{~kJ} / \mathrm{mol}$ respectively, both of the energies being much greater than the averaged one of emitted electrons mentioned above. However, some of the emitted electrons are considered to have sufficient 
energy which is necessitated to dissociate $\mathrm{H}-\mathrm{OH}$ and/or $\mathrm{O}-\mathrm{H}$ bond, since the energy height during the progress of fatigue shows such a broad distribution as is shown in Fig. 9. Consequently, the environmental influence of water vapor in atmosphere on fatigue of metals may be explained by the following process; atomic hydrogen originating from dissociated water vapor in the atmosphere by exo-electrons is occluded into metals, which results in hydrogen embrittlement with the progress of stress cycles.

Broom and Nicholson have reported that the very small amount of water vapor does shorten fatigue lives of a high-strength aluminum alloy, whilst $\mathrm{H}_{2}$ and $\mathrm{O}_{2}$ dried by a liquid nitrogen trap increase the fatigue lives ${ }^{(9)}$. Since oxygen is indispensable to the exoelectron emission ${ }^{(2)}$ and the emitted electrons increase markedly with the increase of water vapor pressure ${ }^{(10)}$, the experimental result that no damaging effect of dry hydrogen was observed as mentioned above may be explained by exo-electron emission.

4. Relation between counted rate and repeated stress amplitude

Figure 10 illustrates a relation between the counted rate and stress amplitude varied stepwise at cycle numbers of $n=5.8 \times 10^{5}$ where the rate is almost constant. A straight line is obtained between the counted rate on a logarithmic scale and stress amplitude. The counted rate increases markedly with increasing stress amplitude. The emission

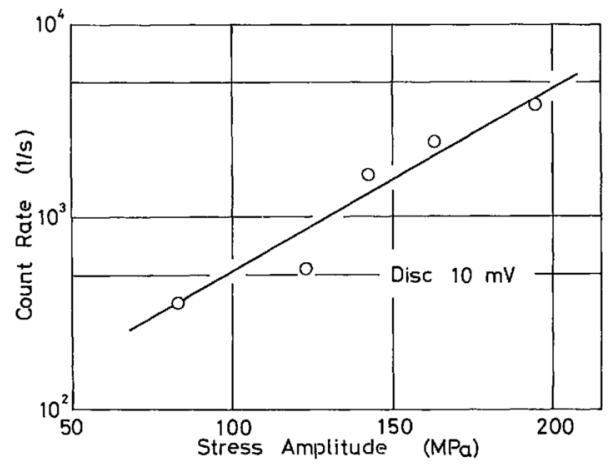

Fig. 10 Relation between count rate per sec and applied stress amplitude. could not be counted under a stress smaller than $\sigma_{0}=80 \mathrm{MPa}$ because of the resonance characteristics of the fatigue testing machine. Hempel and others ${ }^{(3)}$ reported threshold values for the electron emission from aluminum, which were the strain of $1 \%$ in tensile deformation and the stress amplitude of $20 \mathrm{MPa}$ in repetitions of pulsating tensile stress (fatigue strength at $10^{7}=40 \mathrm{MPa}$ ). However, the above results cannot prove the existence of a threshold stress (strain) for the electron emission, since the counted rate, which was on the order of $10^{4}$ count/min, is much smaller than that in the present experiment in spite of an illuminated condition, and a noise was also rather high.

\section{Relation between counted rate and stress cycle frequency}

Figure 11 illustrates the counted rate per min at various cycle frequencies under the reversed stress $\sigma_{0}=176 \mathrm{MPa}$, where the counted electrons as a background is substracted from the emission rate. It is known that the rate depends markedly upon stress cycle frequency, abruptly increasing with increasing cycle frequency to a value of $1.6 \times 10^{5} \mathrm{count} / \mathrm{min}$ at $14.5 \mathrm{~Hz}$. The relation between the counted rate per cycle and the stress cycle frequency is shown in Fig. 12., which illustrates a much
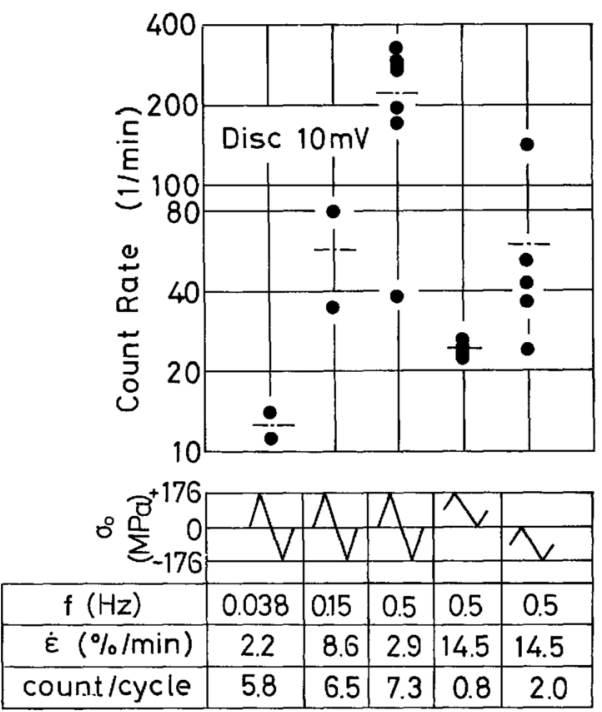

Fig. 11 Relation between count rate per min and stress cycle frequency. 


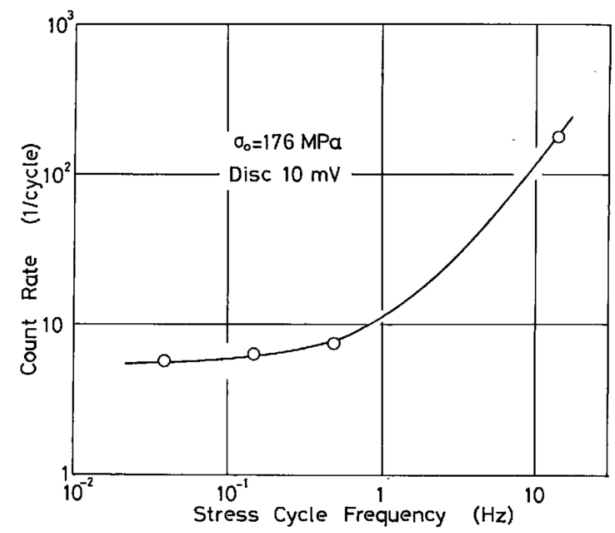

Fig. 12 Relation between count rate per cycle and stress cycle frequency.

increased counted rate per cycle at a higher cycle frequency. Judging from the emission characteristics, it can be said that the chemical activity per cycle of fatigued surfaces is greater at a higher cycle frequency, since emitted electrons are able to cause a chemical reaction with the environment.

More exo-electrons are emitted under pulsated compressive stress than under pulsated tensile stress, as shown in Fig. 11.

\section{Conclusions}

The exo-electron emission from metal surfaces subjected to repeated stresses has been studied using an electron-multiplier counting system, and the environmental effects on fatigue are discussed from the viewpoint of exo-electron emissions.

(1) A great deal of exo-electrons are emitted from metal surface subjected to repetitional stresses. The exo-electrons sharply increase immediately after a beginning of the fatigue test and take a maximum value. Thereafter the counted rate gradually decreases and becomes almost constant.
(2) The decay of exo-electron emission after termination of stress repetitions is very fast, its half life being shorter than $1 \mathrm{~s}$. The decay is different at cycle numbers before and after $N_{\text {emax }}$ at which the emission rate takes a maximum, and the decay is very fast at the beginning of fatigue cycles. The counted rate after a duration longer than 10 20 s decreases linearly on logarithmic scales, its slope being constant, -0.33 . The slope $(-0.33)$ is much smaller than that $(-1)$ of the decay from a mechanically processed surface, which shows the more persistent emission characteristic of a surface subjected to fatigue damage than the emission of a mechanically processed surface.

(3) The pulse height distribution of emitted electrons is independent of the progress of fatigue damage. However, sufficient allowance should be made for the role of exo-electrons in the environmental effect on fatigue strength, in view of the energy height of exo-electrons.

(4) The counted rate per cycle markedly depends upon stress cycle frequency, being abruptly increased with increasing cycle frequency.

\section{REFERENCES}

(1) J. Kramer: Z. Phys., 125 (1949), 739.

(2) L. Lohff: Z. Phys., 146 (1956), 436.

(3) M. Hempel, A. Kochendörfer und A. Tietze: Arch. Eisenhüttenw., 35 (1964), 465.

(4) J. F. Moore, S. Tsang and G. Martin: U.S. Technical Report AFMR-TR-71-185, (1971).

(5) W. J. Baxter: Met. Trans., 6A (1975), 749.

(6) L. Grunberg: Proc. Phys. Soc. Lond., Ser. B, 66 (1953), 153.

(7) J. C. Grosskreutz: J. Appl. Phys., 33 (1962), 2653.

(8) N. Sato: Bull. Japan Inst. Metals (in Japanese), 7 (1968), 313.

(9) T. Broom and A. Nicholson: J. Inst. Metals, 89 (1961), 183.

(10) J. A. Ramsey: Surface Science, 8 (1967), 313. 